\title{
CONTINUOUS FAMILIES OF RIEMANNIAN MANIFOLDS, ISOSPECTRAL ON FUNCTIONS BUT NOT ON 1-FORMS.
}

\author{
Ruth Gornet
}

\begin{abstract}
The purpose of this paper is to present the first continuous families of Riemannian manifolds isospectral on functions but not on 1-forms, and simultaneously, the first continuous families of Riemannian manifolds with the same marked length spectrum but not the same 1-form spectrum. The examples presented here are Riemannian three-step nilmanifolds and thus provide a counterexample to the Ouyang-Pesce Conjecture for higher-step nilmanifolds. Ouyang and Pesce independently showed that all isospectral deformations of two-step nilmanifolds must arise from the Gordon-Wilson method for constructing isospectral nilmanifolds. In particular, all continuous families of Riemannian two-step nilmanifolds that are isospectral on functions must also be isospectral on $p$-forms for all $p$. They conjectured that all isospectral deformations of nilmanifolds must arise in this manner. These examples arise from a general method for constructing isospectral Riemannian nilmanifolds previously introduced by the author.
\end{abstract}

\section{Section 1. Introduction.}

The spectrum of a closed Riemannian manifold $(M, g)$, denoted $\operatorname{spec}(M, g)$, is the collection of eigenvalues with multiplicities of the associated LaplaceBeltrami operator acting on smooth functions. Two Riemannian manifolds $(M, g)$ and $\left(M^{\prime}, g^{\prime}\right)$ are said to be isospectral if $\operatorname{spec}(M, g)=\operatorname{spec}\left(M^{\prime}, g^{\prime}\right)$. The Laplace-Beltrami operator may be extended to act on smooth $p$-forms by $\Delta=d \delta+\delta d$, where $\delta$ is the metric adjoint of $d$. We denote its eigenvalue spectrum by $p$-spec $(M, g)$.

This paper addresses the question of spectral rigidity: "If we continuously deform a manifold while keeping the Laplace spectrum constant, must the resulting manifolds be isometric?" That is, can we determine the geometry of a manifold based on nearby behavior of the spectrum?

1991 Mathematics Subject Classification. Primary 58G25, 22E27; Secondary 53C30, 53C22.

Key words and phrases. Isospectral manifolds; Laplacian on forms; Nilmanifolds; Isospectral deformations; Marked length spectrum.

Research at Texas Tech University supported in part by a State of Texas, Advanced Research Program Grant. 
Sometimes the answer is "Yes." Croke and Sharafutdinov [CS], generalizing work of Guillemin and Khazdan $[\mathrm{GuK}]$ for surfaces, have recently showed spectral rigidity of negatively curved manifolds. Flat tori are known to be spectrally rigid [Wp], while Heisenberg manifolds [P1] are spectrally rigid within the family of left invariant metrics.

Nilmanifolds and nilpotent Lie groups have played a vital role in showing that the general answer is "No." In 1984, Gordon and Wilson [GW1] constructed the first nontrivial isospectral deformations, continuous families of isospectral, nonisometric Riemannian nilmanifolds. The Gordon-Wilson method has been modified to produce conformally equivalent isospectral deformations of Riemannian two-step nilspaces [DG2], [BG], and nontrivial isospectral deformations arbitrarily close to Heisenberg manifolds [Sch2]. Using new techniques, Gordon and Wilson [GW3] have recently used nilmanifolds to construct continuous families of isospectral (Dirichlet and Neumann) manifolds with boundary that are nonlocally isometric. These are the only known examples of nontrivial isospectral deformations.

While two-step nilmanifolds are not spectrally rigid, they do satisfy a "modified" rigidity. In 1992, Ouyang [O] and Pesce [P3], independently showed that isospectral deformations of two-step nilmanifolds must arise from the GordonWilson method. Ouyang and Pesce conjectured, "isospectral deformations of nilmanifolds must arise from the Gordon-Wilson method." It was expected that a combination of their different techniques would prove the result on higher-step nilmanifolds.

In 1985, Sunada [Su] gave an elegant method for constructing pairs of isospectral manifolds that can be explained solely in representation theoretic terms. (See [B2] for a discussion.) His method was later generalized [DG2] to include the Gordon-Wilson deformations. Sunada's method and its generalizations produce manifolds that are strongly isospectral, that is, they must share the same spectrum for all natural, strongly elliptic operators. In particular, isospectral deformations arising from the Gordon-Wilson method must share the same $p$-form spectrum for all $p$. Conversely, Schueth [Sch1] showed that if one uses representation theoretic techniques alone to obtain continuous families of isospectral nilmanifolds, then the deformation must arise from the Gordon-Wilson method.

As most constructions for producing families of isospectral manifolds can by explained by Sunada's method or its generalizations, examples of Riemannian manifolds that are isospectral on functions but not on forms are sparse. Gordon [G2] has constructed pairs of Heisenberg manifolds that are isospectral on functions but not on 1-forms, and the author [Gt3,4] has constructed pairs of three-step Riemannian nilmanifolds with this property. The only other known examples of manifolds that are isospectral on functions but not on $p$-forms for all $p$ are lens spaces. For any choice of $P \in \mathbb{Z}^{+}$, Ikeda [I2] has constructed examples of pairs isospectral lens spaces that are isospectral on $p$-forms for 
$p=0,1, \ldots, P$ but not isospectral on $(P+1)$-forms. Recently, Pesce $[\mathrm{P} 4]$ has been able to explain the Ikeda examples in a Sunada-like setting, which requires a genericity assumption excluding nilmanifolds. Nilmanifolds, therefore, now account for all known isospectral manifolds that do not fall under a Sunada-like setting.

The main result of this paper is the following.

Theorem: There exist continuous families of Riemannian manifolds that are isospectral on functions but not on 1-forms.

The examples presented here are three-step nilmanifolds, and thus provide a counterexample to the Ouyang-Pesce conjecture. The new deformations arise from a general method for constructing isospectral Riemannian nilmanifolds introduced in [Gt4], which combines techniques from representation theory and Riemannian geometry.

All known examples of strongly isospectral nilmanifolds arise from the GordonWilson method, leading to the following.

Conjecture: Continuous families of nilmanifolds that are isospectral on functions and on 1-forms must arise from the Gordon-Wilson method.

Two Riemannian manifolds $\left(M_{1}, g_{1}\right)$ and $\left(M_{2}, g_{2}\right)$ have the same marked length spectrum if there exists an isomorphism between the fundamental groups of $M_{1}$ and $M_{2}$ such that corresponding free homotopy classes of loops contain smoothly closed geodesics of the same length. (See Section 5 for more details.)

The relationship between the Laplace spectrum and lengths of closed geodesics arises from the study of the wave equation (see [DGu]), and in the case of compact, hyperbolic manifolds, from the Selberg Trace Formula (see [C, Chapter $\mathrm{XI}]$ ). Colin de Verdière [CdV] has shown that generically, the Laplace spectrum determines the lengths of closed geodesics. All known examples of manifolds that are isospectral have the same lengths of closed geodesics.

Croke [Cr] and Otal [Ot12] independently showed that if a pair of compact surfaces with negative curvature have the same marked length spectrum, they are necessarily isometric. This is due to the fact that in certain cases, the marked length spectrum and the geodesic flow are (roughly) equivalent notions. Gordon, Mao, and Schueth [GM1,2], [GMS] have shown that generically, twostep nilmanifolds with conjugate geodesic flows must be isometric.

Isospectral Riemannian manifolds constructed using the Gordon-Wilson method necessarily have the same marked length spectrum, while Eberlein [E] proved the converse in the case of two-step nilmanifolds. Thus the GordonWilson method produces continuous families of strongly isospectral Riemannian manifolds with the same marked length spectrum. In contrast, we prove the following.

Theorem: There exist continuous families of Riemannian manifolds 
with the same marked length spectrum but not the same spectrum on 1-forms.

Discrete families of manifolds with this property were presented in [Gt4], to which the reader is referred for an introduction to the relationships among the marked length spectrum, the length spectrum, the Laplace spectrum on functions, and the Laplace spectrum on forms on discrete families of Riemannian manifolds. The only other known examples of manifolds that have the same marked length spectrum but not the same spectrum on 1-forms are the Zoll spheres and standard spheres, which are not even isospectral on functions.

This paper is organized as follows. We briefly review background material and the Gordon-Wilson method in Section 2. The techniques used to construct the new examples are discussed in Section 3. One way to show that isospectral nilmanifolds cannot arise from the Gordon-Wilson method is by showing that no diffeomorphism between the manifolds of the correct type can exist. This approach is discussed in Section 3, as it lends insight into the types of Riemannian nilmanifolds that can support isospectral deformations that are not strongly isospectral. The new examples are presented in Section 4, while the marked length spectrum and the 1-form spectrum are the focus of Sections 5 and 6 , respectively.

Acknowledgments. The author wishes to thank the joint NSF-CNRS program in Spectral Geometry for their support of the workshop at Dartmouth in February 1996, where much of this work was carried out. The author also wishes to thank Ed Wilson and David L. Webb for insightful conversations.

\section{Section 2. Background and Notation.}

2.1 Riemannian Nilmanifolds. Let $G$ be a simply connected Lie group, and let $\Gamma$ be a cocompact, discrete subgroup of $G$. A Riemannian metric $g$ is left invariant if the left translations of $G$ are isometries. The left invariant metric $g$ descends to a Riemannian metric on $\Gamma \backslash G$, also denoted by $g$. Note that a left invariant metric is determined by specifying an orthonormal basis $\left\{E_{1}, \cdots, E_{n}\right\}$ of the Lie algebra $\mathfrak{g}$ of $G$. As $G$ is unimodular, the LaplaceBeltrami operator $\Delta$ of $(\Gamma \backslash G, g)$ is just

$$
\Delta=-\sum_{i=1}^{n} E_{i}{ }^{2} .
$$

For a Lie algebra $\mathfrak{g}$, denote by $\mathfrak{g}^{(1)}$ the derived algebra $[\mathfrak{g}, \mathfrak{g}]$ of $\mathfrak{g}$. Inductively, define $\mathfrak{g}^{(k+1)}=\left[\mathfrak{g}, \mathfrak{g}^{(k)}\right]$. A Lie algebra $\mathfrak{g}$ is k-step nilpotent if $\mathfrak{g}^{(k-1)}$ is nontrivial and central. A Lie group $G$ is $k$-step nilpotent if its Lie algebra is. Let $\Gamma$ be a cocompact, discrete subgroup of a simply connected nilpotent Lie group $G$ with left invariant metric $g$. The locally homogeneous space $(\Gamma \backslash G, g)$ is called a Riemannian nilmanifold.

For more details about nilmanifolds and nilpotent Lie groups see [CG]. 
2.2 Isometries of Nilmanifolds. We write $(M, g)=\left(M^{\prime}, g^{\prime}\right)$ if the Riemannian manifolds $(M, g)$ and $\left(M^{\prime}, g^{\prime}\right)$, are isometric. Let $(\Gamma \backslash G, g)$ be a Riemannian nilmanifold. Any automorphism $\Phi \in \operatorname{Aut}(G)$ induces an isometry from $\left(\Gamma \backslash G, \Phi^{*} g\right)$ to $(\Phi(\Gamma) \backslash G, g)$. Any inner automorphism $I_{x} \in \operatorname{IA}(G)$ induces an isometry from $(\Gamma \backslash G, g)$ to $\left(\Gamma \backslash G, I_{x}^{*} g\right)$ via the mapping $R_{x}: \Gamma \backslash G \rightarrow \Gamma \backslash G$ defined by $R_{x}(\Gamma y)=\Gamma y x$. Let $\operatorname{OA}(G, g)=\left\{\Upsilon \in \operatorname{Aut}(G): \Upsilon^{*} g=g\right\}$, the orthogonal automorphisms of $G$ with respect to $g$. If $\Upsilon$ is in $\operatorname{OA}(G, g)$, then $(\Gamma \backslash G, g)=\left(\Gamma \backslash G, \Upsilon^{*} g\right)$.

Recall that for any Lie group $G$ with Lie algebra $\mathfrak{g}$, Aut $(\mathfrak{g})$ is a Lie group with Lie algebra $\operatorname{Der}(\mathfrak{g})$. We denote by $\exp : \operatorname{Der}(\mathfrak{g}) \rightarrow \operatorname{Aut}(\mathfrak{g})$ the Lie algebra exponential, which reduces to the matrix exponential by looking at matrix representations.

2.3 Notation. For every $D \in \operatorname{Der}(\mathfrak{g})$, we associate an operator $D^{*}$ that takes the two-form $\omega$ to the two-form defined by $D^{*} \omega(X, Y)=\omega(D X, Y)+$ $\omega(X, D Y)$.

One generally denotes by $\Phi^{*} \omega$ the two-form defined by $\Phi^{*} \omega(X, Y)=\omega\left(\Phi_{*}(X), \Phi_{*}(Y)\right)$, where $\Phi_{*} \in \operatorname{Aut}(\mathfrak{g})$. As $D \in \operatorname{Der}(\mathfrak{g})$ is the derivative of a curve $\Phi_{s *}$ in $\operatorname{Aut}(\mathfrak{g})$, $D^{*} \omega$ is the derivative of $\Phi_{s}^{*} \omega$ in the obvious sense.

With this notation, $\mathrm{OA}(G, g)$ is a Lie group with Lie algebra $\mathrm{OD}(G, g)=$ $\left\{D \in \operatorname{Der}(\mathfrak{g}): D^{*} g=0\right\}$, the skew-symmetric derivations of $\mathfrak{g}$.

2.4 Almost Inner Automorphisms [GW1], [G1]. Let $G$ be a Lie group with Lie algebra $\mathfrak{g}$ and cocompact, discrete subgroup $\Gamma$. A Lie group automorphism $\Psi$ of $G$ is almost inner if for all $x$ in $G$, the elements $x$ and $\Psi(x)$ are conjugate in $G$. The automorphism $\Psi$ of $G$ is $\Gamma$-almost inner if for all $\gamma$ in $\Gamma$, the elements $\gamma$ and $\Psi(\gamma)$ are conjugate in $G$. A Lie algebra isomorphism $\Psi_{*}$ is almost inner (resp., $\Gamma$-almost inner) if for every $X$ in $\mathfrak{g}$, (resp., $X$ in $\log \Gamma$ ), $\Psi_{*}(X)=A d(x)(X)$ for some $x$ in $\mathfrak{g}$. Here Ad denotes the adjoint representation of $G$ on $\mathfrak{g}$. A derivation $D$ is inner if there exists $Y$ in $\mathfrak{g}$ such that $D=\operatorname{ad}(Y)$. A derivation $D$ is almost inner, (resp., $\Gamma$-almost inner) if for every $X$ in $\mathfrak{g}$ (resp., $X$ in $\log \Gamma$ ) there exists $Y$ in $\mathfrak{g}$ such that $D(X)=\operatorname{ad}(Y)(X)$.

Denote by $\operatorname{IA}(G)$, (resp., $\operatorname{AIA}(G), \operatorname{AIA}(G ; \Gamma))$ the set of inner (resp., almost inner, $\Gamma$-almost inner) automorphisms of $G$. Note that $\operatorname{IA}(G) \subset \operatorname{AIA}(G) \subset$ $\operatorname{AIA}(G ; \Gamma)$, and there exist examples [Gt1], [GW1] demonstrating that each of these containments can be strict. Define $\operatorname{IA}(\mathfrak{g}), \operatorname{AIA}(\mathfrak{g}), \operatorname{AIA}(\mathfrak{g} ; \Gamma), \operatorname{ID}(\mathfrak{g})$, $\operatorname{AID}(\mathfrak{g})$, and $\operatorname{AID}(\mathfrak{g} ; \Gamma)$ analogously.

If $G$ is nilpotent, then $\Psi \in \operatorname{Aut}(G)$ is inner (resp., almost inner, $\Gamma$-almost inner) if and only if $\Psi_{*} \in \operatorname{Aut}(\mathfrak{g})$ is inner (resp., almost inner, $\Gamma$-almost inner). Also, $\operatorname{IA}(\mathfrak{g}), \operatorname{AIA}(\mathfrak{g})$, and $\operatorname{AIA}(\mathfrak{g} ; \Gamma)$ are connected Lie subgroups of $\operatorname{Aut}(\mathfrak{g})$, with Lie algebras $\operatorname{ID}(\mathfrak{g}), \operatorname{AID}(\mathfrak{g})$, and $\operatorname{AID}(\mathfrak{g} ; \Gamma)$, respectively.

2.5 Theorem [GW1], [G2]. Let $\Gamma$ be a cocompact, discrete subgroup of a 
simply connected, nilpotent Lie group $G$ with left invariant metric $g$.

(1) If $\Psi$ is a $\Gamma$-almost inner automorphism, then $p$-spec $(\Gamma \backslash G, g)=p-\operatorname{spec}(\Psi(\Gamma) \backslash G, g)$ for $p=0,1, \cdots, \operatorname{dim}(G)$.

(2) If $\Psi_{s}$ is a continuous family of $\Gamma$-almost inner automorphisms that are not inner automorphisms, then $\left(\Psi_{s}(\Gamma) \backslash G, g\right)=\left(\Gamma \backslash G, \Psi_{s}^{*} g\right)$ is a nontrivial, isospectral deformation.

(3) There exist two-step nilpotent Lie groups supporting continuous families of $\Gamma$-almost inner automorphisms that are not inner automorphisms.

Conversely, Ouyang and Pesce independently proved the following.

2.6 Theorem [O],[OP],[P2]. Let $\left(\Gamma \backslash G, g_{s}\right)$ be a continuous family of Riemannian two-step nilmanifolds that are isospectral on functions. Then there exists a continuous family $\Psi_{s}$ of $\Gamma$-almost inner automorphisms with $\Psi_{0}=I$ such that $g_{s}=\Psi_{s}^{*} g_{0}$.

2.7 Notation and Remarks. Define

$\operatorname{GW}(G, g ; \Gamma)=\mathrm{OA}(G, g) \circ \operatorname{AIA}(G ; \Gamma)=\{\Upsilon \circ \Psi: \Upsilon \in \mathrm{OA}(G, g), \Psi \in \operatorname{AIA}(G ; \Gamma)\}$,

$\operatorname{GW}(G, g)=\mathrm{OA}(G, g) \circ \operatorname{AIA}(G)=\{\Upsilon \circ \Psi: \Upsilon \in \mathrm{OA}(G, g), \Psi \in \operatorname{AIA}(G)\}$.

The group $\operatorname{AIA}(G)$ is a normal subgroup of $\operatorname{Aut}(G)$, so $\operatorname{GW}(G, g)$ is a Lie group with Lie algebra $\mathrm{OD}(\mathfrak{g}, g) \oplus \operatorname{AID}(\mathfrak{g})$. In particular, if $D \in \mathrm{OD}(\mathfrak{g}, g) \oplus$ $\operatorname{AID}(\mathfrak{g})$, then the Lie group automorphism $\Phi$ defined at the Lie algebra level by $\Phi_{*}=\exp (D)$ is in $\operatorname{GW}(G, g)$. A priori, the set $\mathrm{GW}(G, g ; \Gamma)$ is not a subgroup of $\operatorname{Aut}(G)$, as there is no reason to assume that $\operatorname{AIA}(G ; \Gamma)$ is a normal subgroup of $\operatorname{Aut}(G)$, although this proves to be the case in all known examples.

For the nilpotent Lie groups studied in Section $4, \operatorname{AIA}(G)=\operatorname{AIA}(G ; \Gamma)$ for any choice of cocompact, discrete subgroup $\Gamma$ of $G$.

2.8 Proposition [GW1]. Let $G$ be a simply connected, nilpotent Lie group with cocompact discrete subgroup $\Gamma$ and left invariant metric $g$. Let $\Phi_{s}$ be a continuous family of automorphisms of $G$ such that $\Phi_{0}$ is the identity.

(1) The continuous family $\left(\Gamma \backslash G, \Phi_{s}^{*} g\right)$ is trivial if and only if $\Phi_{s} \in O A(G, g) \circ$ $I A(G)$ for all $s$.

(2) The continuous family $\left(\Gamma \backslash G, \Phi_{s}^{*} g\right)$ is Gordon-Wilson if and only if there exists $\Upsilon$ in $O A(G, g)$ such that for all $s, \Phi_{s} \circ \Upsilon \in G W(G, g ; \Gamma)$.

Note that by $(2.7)$, if $\mathrm{GW}(G ; \Gamma)$ is a group, then we may assume $\Upsilon=I$ in the statement of Proposition 2.8.

Comments on Proof. This follows directly from [GW1, Corollary 5.3], using $\mathcal{D}=\{\delta \in \operatorname{Aut}(G): \delta(\Gamma)=\Gamma\}$ discrete and $\Phi_{0}$ the identity. Note that the group defined by Gordon-Wilson as $\mathrm{K}_{g}$ is denoted here by $\mathrm{OA}(G, g)$. 
2.9 Quotient Nilmanifolds. Let $G$ be a simply connected, $k$-step nilpotent Lie group with Lie algebra $\mathfrak{g}$. Define $\bar{G}$ to be the simply connected, $(k-1)$-step nilpotent Lie group $\bar{G}=G / G^{(k-1)}$. For $\Gamma$ a cocompact, discrete subgroup of $G$, denote by $\bar{\Gamma}$ the image of $\Gamma$ under the canonical projection from $G$ onto $\bar{G}$. The group $\bar{\Gamma}$ is then a cocompact, discrete subgroup of $\bar{G}$. We denote elements of $\bar{G}$ by $\bar{x}$, where $\bar{x}$ is the image of $x$ under the canonical projection from $G$ onto $\bar{G}$. Note that $\bar{x}=\bar{y}$ if and only if $x y^{-1}$ is in $G^{(k-1)} \subset Z(G)$. If $\Phi \in \operatorname{Aut}(G)$, we denote by $\bar{\Phi} \in \operatorname{Aut}(\bar{G})$ the projection of $\Phi$ onto $\bar{G}$. The Lie algebra $\overline{\mathfrak{g}}$ of $\bar{G}$ is just $\mathfrak{g} / \mathfrak{g}^{(k-1)}$. We denote elements of $\overline{\mathfrak{g}}$ by $\bar{X}$, where $\bar{X}$ is the image of $X$ under the canonical projection from $\mathfrak{g}$ onto $\overline{\mathfrak{g}}$.

For a left invariant metric $g$ on $G$, we associate a left invariant metric $\bar{g}$ on $\bar{G}$ by restricting $g$ to an orthogonal complement of $\mathfrak{g}^{(k-1)}$ in $\mathfrak{g}$. We call the $(k-1)$-step nilmanifold $(\bar{\Gamma} \backslash \bar{G}, \bar{g})$ the quotient nilmanifold of $(\Gamma \backslash G, g)$. Using the definition of $\bar{g}$, one easily sees that the projection $(\Gamma \backslash G, g) \rightarrow(\bar{\Gamma} \backslash \bar{G}, \bar{g})$ is a Riemannian submersion with totally geodesic fibers.

2.10 Definition. The simply connected nilpotent Lie group $G$ is strictly nonsingular if for every noncentral $x$ in $G$, if $\bar{x}=\bar{v}$ then $x$ and $v$ are conjugate in $G$. Restated, for every noncentral $x$ in $G$ and every $z$ in $Z(G)$, there exists $y$ in $G$ such that $y x y^{-1}=x z$. The nilpotent Lie algebra $\mathfrak{g}$ is strictly nonsingular if for every noncentral $X$ in $\mathfrak{g}$ and every $Z$ in $\mathfrak{z}$ there exists $Y$ in $\mathfrak{g}$ such that $[X, Y]=Z$. That is, for every noncentral $X$ in $\mathfrak{g}, \mathfrak{z} \subset \operatorname{ad}(X)(\mathfrak{g})$.

\subsection{Remarks.}

(1) One easily checks that these notions are equivalent, i.e., a nilpotent Lie group is strictly nonsingular if and only if its Lie algebra is strictly nonsingular.

(2) Equivalently, a nilpotent Lie group $G$ is strictly nonsingular if and only if every fiber of the submersion $G \rightarrow \bar{G}$ is contained in a single orbit of the action of $G$ on itself by conjugation. A two-step nilpotent Lie group is strictly nonsingular if and only if every fiber is equal to an orbit.

(3) If $G$ is strictly nonsingular, then $Z(G)=G^{(k-1)}$, but not conversely.

(4) The examples presented in Section 4 show that one can have both $G$ and $\bar{G}$ strictly nonsingular, but this is not the case in general. See $[\mathrm{Gt} 1,2]$ for examples.

\section{Section 3. New Deformations.}

The method we will use to construct the new isospectral deformations is the following. This method has previously produced discrete families of Riemannian manifolds, isospectral on functions but not on 1-forms [G2], [Gt3,4].

3.1 Theorem [Gt2, Theorem 3.3]. Let $G$ be a simply connected, strictly nonsingular nilpotent Lie group with left invariant metric $g$. If $\Gamma_{1}$ and $\Gamma_{2}$ are 
cocompact, discrete subgroups of $G$ such that

$$
\Gamma_{1} \cap Z(G)=\Gamma_{2} \cap Z(G) \quad \text { and } \operatorname{spec}\left(\bar{\Gamma}_{1} \backslash \bar{G}, \bar{g}\right)=\operatorname{spec}\left(\bar{\Gamma}_{2} \backslash \bar{G}, \bar{g}\right),
$$

then $\operatorname{spec}\left(\Gamma_{1} \backslash G, g\right)=\operatorname{spec}\left(\Gamma_{2} \backslash G, g\right)$.

3.2 Remark. Any deformation using Theorem 3.1 must be of the form $\left(\Gamma_{s} \backslash G, g\right)$, where $\Gamma_{s}$ depends continuously on $s$. In particular, the manifolds must have a common Riemannian covering $(G, g)$, hence are locally isometric. It is known [Sch1] that if $\Gamma_{s}$ is any continuous family of cocompact, discrete subgroups of a nilpotent Lie group $G$, then there exists a continuous family of automorphisms $\Phi_{s} \in \operatorname{Aut}(G)$ with $\Phi_{0}=I$ such that $\Gamma_{s}=\Phi_{s}(\Gamma)$. So the deformations arising from Theorem 3.1 are of the form $\left(\Gamma \backslash G, \Phi_{s}^{*} g\right), \Phi_{s} \in \operatorname{Aut}(G)$.

3.3 Corollary. Let $G$ be a strictly nonsingular nilpotent Lie group with left invariant metric $g$ and cocompact, discrete subgroup $\Gamma$. Assume there exists a continuous family of automorphisms $\Phi_{s} \in$ Aut $(G), \Phi_{0}=I$, such that for all $s$

(1) $\Phi_{s}$ restricted to $Z(G)$ is the identity, and

(2) $\bar{\Phi}_{s} \in G W(\bar{G}, \bar{g} ; \bar{\Gamma})$.

Then $\left(\Gamma \backslash G, \Phi_{s}^{*} g\right)$ is an isospectral deformation. The deformation is trivial if and only if $\Phi_{s} \in O A(G, g) \circ I A(G)$ for all s. The deformation is Gordon-Wilson if and only if there exists $\Upsilon \in O A(G, g)$ such that $\Phi_{s} \circ \Upsilon \in G W(G, g ; \Gamma)$ for all $s$.

Note again that by $(2.7)$, if $\mathrm{GW}(G, g ; \Gamma)$ is a group, then we may assume $\Upsilon=I$ in the statement of Corollary 3.3.

Proof. This follows directly from Theorem 3.1, Theorem 2.5, and Proposition 2.8 .

To use Corollary 3.3 to obtain isospectral deformations that are not GordonWilson, we exhibit a strictly nonsingular, three-step nilpotent Lie group $G$ and a continuous family of automorphisms $\Phi_{s} \in \operatorname{Aut}(G), \Phi_{s} \notin \mathrm{GW}(G, g)$ such that $\bar{\Phi}_{s} \in \operatorname{GW}(\bar{G}, \bar{g})$. For the examples presented here, $\operatorname{AIA}(G ; \Gamma)=\operatorname{AIA}(G)$ and $\operatorname{AIA}(\bar{G} ; \bar{\Gamma})=\operatorname{AIA}(\bar{G})$ for any choice of cocompact discrete subgroup $\Gamma$ of $G$.

3.4 Remark. The key to the new deformations is close study of the behavior of the orthogonal automorphisms on the quotient nilmanifold. Example I below exhibits a nontrivial isospectral deformation where almost inner automorphisms play no role whatsoever, i.e., $\mathrm{GW}(\bar{G}, \bar{g} ; \bar{\Gamma})$ may be replaced by $\operatorname{OA}(\bar{G}, \bar{g})$ in condition (2) of Corollary 3.3. So the isospectral deformation $\Phi_{s}$ projects to a trivial deformation on the quotient nilmanifold, but $\Phi_{s}$ is nontrivial. If we disregard $\mathrm{OA}(\bar{G}, \bar{g})$, however, we reduce to the case of Gordon-Wilson deformations. For if we let $\Phi_{s}$ satisfy the conditions of Corollary 3.3 and let $\bar{\Phi}_{s} \in \operatorname{AIA}(\bar{G} ; \bar{\Gamma})$ for all $s$, then the elements $\bar{\gamma}$ and $\bar{\Phi}_{s}(\bar{\gamma})$ are conjugate for all $\bar{\gamma} \in \bar{\Gamma}$. By strict nonsingularity, $\gamma$ and $\Phi_{s}(\gamma)$ are necessarily conjugate, and the deformation is Gordon-Wilson. 
3.5 Remark. The geometric role of the orthogonal automorphisms is as follows. Fix an orthonormal basis of the $k$-dimensional center $\mathfrak{z}$ of $\mathfrak{g}$ and extend it to a $(k+n)$-dimensional orthonormal basis $\mathcal{B}$ of $\mathfrak{g}$. If $\Phi_{s}$ satisfies the hypotheses of Corollary 3.3, then $\bar{\Phi}_{s}=\Upsilon_{s} \circ \Psi_{s}$, where $\Upsilon_{s} \in \mathrm{OA}(\bar{G}, \bar{g})$ and $\Psi_{s} \in \operatorname{AIA}(\bar{G}, \bar{\Gamma})$. View $\Upsilon_{s *}$ as an element of $O(n)$ by considering its matrix representation with respect to $\mathcal{B}$. Extend $\Upsilon_{s *}$ to $\hat{\Upsilon}_{s *} \in O(n+k)$ by the $k \times k$ identity matrix:

$$
\hat{\Upsilon}_{s *}=\left(\begin{array}{cc}
\Upsilon_{s *} & 0 \\
0 & I_{k}
\end{array}\right)
$$

The elements of the group $O(n+k)$, hence the matrices $\hat{\Upsilon}_{s *}$, act on our metric $g$ by taking the orthonormal basis $\mathcal{B}$ of $\mathfrak{g}$ to $\hat{\Upsilon}_{s *}^{-1} \mathcal{B}$, which determines the same left invariant metric $g$ of $G$. So with the obvious notation, we have

$$
\left(\Gamma \backslash G, \Phi_{s}^{*} g\right)=\left(\Gamma \backslash G, \hat{\Psi}_{s}^{*} \circ \hat{\Upsilon}_{s}^{*} g\right)=\left(\Gamma \backslash G, \hat{\Psi}_{s}^{*} g\right) .
$$

Here $\hat{\Psi}_{s *}=\hat{\Upsilon}_{s *}^{-1} \circ \Phi_{s}$, a triangular matrix with 1's down the diagonal, so $\hat{\Psi}_{s *} \in \operatorname{SL}(n+k)$. Note that if $\mathfrak{g}$ is strictly nonsingular, then $\hat{\Psi}_{s *}$ and $\hat{\Upsilon}_{s *}$ are Lie algebra automorphisms if and only if the deformation is Gordon-Wilson.

The matrices $\hat{\Psi}_{s *}$ deform the orthonormal basis, i.e., the metric $\hat{\Psi}_{s}^{*} g$ is determined by the orthonormal basis $\hat{\Psi}_{s *}^{-1} \mathcal{B}$. If the deformation is not GordonWilson, then the role of $\hat{\Upsilon}_{s}$ is to continuously compensate for $\hat{\Psi}_{s}$ not being an automorphism in a way that does not effect our metric $g$, allowing us to employ Theorem 3.1. On the other hand, assume that we know $\left(\Gamma \backslash G, \hat{\Psi}_{s}^{*} g\right)$ is an isospectral deformation and that the $\hat{\Psi}_{s}$ are not Lie algebra isomorphisms. We might errantly conclude that the manifolds are not locally isometric, i.e., the fact that that the manifolds $\left(\Gamma \backslash G, \hat{\Psi}_{s}^{*} g\right)=\left(\Phi_{s}(\Gamma) \backslash G, g\right)$ are covered by $(G, g)$ is "hidden" by the orthogonal matrices $\hat{\Upsilon}_{s *}$.

We now compute conditions for a Lie algebra automorphism of $\overline{\mathfrak{g}}$ to extend to a Lie group automorphism of the strictly nonsingular Lie algebra $\mathfrak{g}$.

For the remainder, we assume the center $\mathfrak{z}$ of $\mathfrak{g}$ is one-dimensional. Let $G$ be the strictly nonsingular, nilpotent Lie group with Lie algebra $\mathfrak{g}$ and left invariant metric $g$. Let $\mathfrak{N}$ be the orthogonal complement of $\mathfrak{z}$ in $\mathfrak{g}$, so $\mathfrak{g}=\mathfrak{N} \oplus \mathfrak{z}$. Define a closed, nondegenerate two-form $\omega$ on $\mathfrak{N}$ by

$$
[X, Y]=\left([X, Y]_{\mathfrak{N}}, \omega(X, Y) \mathcal{Z}\right)
$$

for all $X, Y \in \mathfrak{g}$. Here $[X, Y]_{\mathfrak{N}}$ denotes the orthogonal projection of $[X, Y]$ onto $\mathfrak{N}$, and $\mathcal{Z}$ is a unit vector in $\mathfrak{z}$. The condition that $\omega$ is closed is equivalent to the Jacobi equations, and the condition that $\omega$ is nondegenerate is equivalent to strict nonsingularity. Note that the vector space $\mathfrak{N}$ with Lie bracket defined by $[,]_{\mathfrak{N}}$ is Lie algebra isomorphic to $\overline{\mathfrak{g}}$. 
3.7 Notation. Conversely, let $(G, g)$ be a $k$-step nilpotent Lie group. Assume there exists a closed, nondegenerate two-form $\omega$ on $\mathfrak{g}$. The central extension of $\mathfrak{g}$ by $\omega$ is the strictly nonsingular, $(k+1)$-step nilpotent Lie algebra $\hat{\mathfrak{g}}$, where $\hat{\mathfrak{g}}=\mathfrak{g} \oplus \mathbb{R} \mathcal{Z}$. The Lie bracket on $\hat{\mathfrak{g}}$ is defined by $(\star)$, that is, $\left[(X, Z),\left(X^{\prime}, Z^{\prime}\right)\right]_{\hat{\mathfrak{g}}}=$ $\left(\left[X, X^{\prime}\right]_{\mathfrak{g}}, \omega\left(X, X^{\prime}\right) \mathcal{Z}\right)$, for all $(X, Z),\left(X^{\prime}, Z^{\prime}\right)$ in $\hat{\mathfrak{g}}$. For $X \in \mathfrak{g}$, we denote by $\hat{X}$ the element $(X, 0)$ in $\hat{\mathfrak{g}}$. We denote by $\hat{G}$ the simply connected nilpotent Lie group with Lie algebra $\hat{\mathfrak{g}}$. Let $g$ be a left invariant metric on $G$. We extend $g$ to a left invariant metric $\hat{g}$ on $\hat{G}$ by requiring that $\mathfrak{g}$ and $\mathcal{Z}$ be orthogonal and that $\mathcal{Z}$ be a unit vector. Note that the quotient Lie group of $(\hat{G}, \hat{g})$ is isometric to the Lie group $(G, g)$.

3.8 Proposition. Let $\mathfrak{g}$ be a nilpotent Lie algebra, and let $\omega$ be a closed, nondegenerate two-form on $\mathfrak{g}$. Let $\hat{\mathfrak{g}}$ be the central extension of $\mathfrak{g}$ by $\omega$.

(1) A derivation $D$ on $\mathfrak{g}$ extends to a derivation $\hat{D}$ on $\hat{\mathfrak{g}}$ if and only if there exists $\eta \in \mathfrak{g}^{*}$ such that $D^{*} \omega=d \eta$, i.e., $D^{*} \omega$ is exact.

(2) A skew-symmetric derivation $S$ on $\mathfrak{g}$ extends to a skew-symmetric derivation $\hat{S}$ on $\hat{\mathfrak{g}}$ if and only if $S^{*} \omega=0$.

3.9 Notation. If $D^{*} \omega=d \eta$ with $\eta \in \mathfrak{g}^{*}$, we denote by $\hat{D}$ the derivation of $\hat{\mathfrak{g}}=\mathfrak{g} \oplus \mathbb{R} \mathcal{Z}$ defined by $\hat{D}(\hat{X})=(D(X),-\eta(X) \mathcal{Z})$ for all $X \in \mathfrak{g}$, and $\hat{D}(\mathcal{Z})=0$.

Proof of 3.8. A straightforward calculation using the definition of a derivation shows that $D$ extends to a derivation $\hat{D}$ on $\hat{\mathfrak{g}}$ if and only if $\hat{D}$ is as in (3.9), and (1) follows. The proof of (2) uses (1) and the definition of $\hat{g}$.

3.10 Corollary. Let $G$ be a strictly nonsingular nilpotent Lie group with Lie algebra $\mathfrak{g}$ and left invariant metric $g$. Assume there exists a closed, nondegenerate two-form $\omega$ on $\mathfrak{g}$ and a derivation $D \in \operatorname{Der}(\mathfrak{g})$ such that

(1) $D=S+A$ where $S \in O D(\mathfrak{g}, g)$ and $A \in A I D(\mathfrak{g})$, and

(2) $D^{*} \omega$ is exact.

Denote by $\hat{D} \in \operatorname{Der}(\hat{\mathfrak{g}})$ the extension of $D$ to $\hat{\mathfrak{g}}$. Define the automorphisms $\Phi_{s} \in$ Aut $(\hat{G})$ on the Lie algebra level by $\Phi_{s *}=\exp (s \hat{D})$. Let $\hat{\Gamma}$ be any cocompact, discrete subgroup of $\hat{G}$. Then $\left(\hat{\Gamma} \backslash \hat{G}, \Phi_{s}^{*} \hat{g}\right)$ is an isospectral deformation. The deformation is trivial if and only if $S^{*} \omega=0$ and $A \in I D(\mathfrak{g})$. The deformation is Gordon-Wilson if and only if $S^{*} \omega=0$.

Proof of 3.10. Follows from Corollary 3.3, Proposition 3.8, and Remark 2.7. Note that by strict nonsingularity, if $\Psi \in \operatorname{Aut}(G)$ and $\bar{\Psi} \in \operatorname{AIA}(\bar{G})$, then $\Psi \in \operatorname{AIA}(G)$.

3.11 Remark. With appropriate additional assumptions, one may extend the above Corollary to include $\operatorname{AID}(\mathfrak{g} ; \Gamma)$. The statement becomes somewhat cumbersome, however, and so is not included here. 
CONTINUOUS FAMILIES OF ISOSPECTRAL RIEMANNIAN MANIFOLDS. 11

\section{Section 4: Examples.}

Consider the strictly nonsingular, two-step nilpotent Lie algebra $\mathfrak{g}$ with orthonormal basis $\left\{X_{1}, X_{2}, X_{3}, X_{4}, Z_{1}, Z_{2}\right\}$ and Lie bracket given by

$$
\begin{aligned}
& {\left[X_{1}, X_{2}\right]=\left[X_{3}, X_{4}\right]=Z_{1},} \\
& {\left[X_{1}, X_{3}\right]=\left[X_{4}, X_{2}\right]=Z_{2},}
\end{aligned}
$$

and all other basis brackets zero. Let $\left\{\alpha_{1}, \alpha_{2}, \alpha_{3}, \alpha_{4}, \zeta_{1}, \zeta_{2}\right\}$ be the dual basis of $\mathfrak{g}^{*}$, and let $G$ be the simply connected Lie group with Lie algebra $\mathfrak{g}$.

\section{Example I.}

Let

$$
\omega=\alpha_{1} \wedge \alpha_{2}+\alpha_{2} \wedge \alpha_{3}+\alpha_{3} \wedge \alpha_{4}+\alpha_{1} \wedge \zeta_{1}-\alpha_{4} \wedge \zeta_{2}
$$

The closed, nondegenerate two-form $\omega$ induces a strictly nonsingular, threestep nilpotent Lie algebra $\hat{\mathfrak{g}}=\mathfrak{g} \oplus \mathbb{R} \mathcal{Z}$ with Lie bracket and left invariant metric as defined in (3.7). So $\hat{\mathfrak{g}}$ is the 7-dimensional Lie algebra with orthonormal basis $\left\{\hat{X}_{1}, \hat{X}_{2}, \hat{X}_{3}, \hat{X}_{4}, \hat{Z}_{1}, \hat{Z}_{2}, \mathcal{Z}\right\}$ and Lie bracket

$$
\begin{aligned}
& {\left[\hat{X}_{1}, \hat{X}_{2}\right]=\left[\hat{X}_{3}, \hat{X}_{4}\right]=\hat{Z}_{1}+\mathcal{Z},} \\
& {\left[\hat{X}_{1}, \hat{X}_{3}\right]=\left[\hat{X}_{4}, \hat{X}_{2}\right]=\hat{Z}_{2},} \\
& {\left[\hat{X}_{2}, \hat{X}_{3}\right]=\left[\hat{X}_{1}, \hat{Z}_{1}\right]=\left[\hat{Z}_{2}, \hat{X}_{4}\right]=\mathcal{Z},}
\end{aligned}
$$

and all other basis brackets zero.

One easily checks that the skew-symmetric derivation $S: \mathfrak{g} \rightarrow \mathfrak{g}$ defined by $X_{1} \rightarrow-X_{4} ; \quad X_{2} \rightarrow 2 X_{3} ; \quad X_{3} \rightarrow-2 X_{2} ; \quad X_{4} \rightarrow X_{1} ; \quad Z_{1} \rightarrow Z_{2} ; \quad Z_{2} \rightarrow-Z_{1}$ satisfies $S^{*} \omega=d \zeta_{2}$, so $S$ extends to the derivation $\hat{S}: \hat{\mathfrak{g}} \rightarrow \hat{\mathfrak{g}}$ given by $\hat{S}(\hat{X})=$ $\left(S(X),-\zeta_{2}(X) \mathcal{Z}\right), \hat{S}(\mathcal{Z})=0$. That is, $\hat{S}$ sends

$\hat{X}_{1} \rightarrow-\hat{X}_{4} ; \quad \hat{X}_{2} \rightarrow 2 \hat{X}_{3} ; \quad \hat{X}_{3} \rightarrow-2 \hat{X}_{2} ; \quad \hat{X}_{4} \rightarrow \hat{X}_{1} ; \quad \hat{Z}_{1} \rightarrow \hat{Z}_{2} ; \quad \hat{Z}_{2} \rightarrow-\hat{Z}_{1}-\mathcal{Z} ; \quad \mathcal{Z} \rightarrow 0$.

Let $\hat{G}$ denote the simply connected, strictly nonsingular nilpotent Lie group with Lie algebra $\hat{\mathfrak{g}}$. Define a continuous family $\Phi_{s}$ of Lie group automorphisms on $\hat{G}$ given at the Lie algebra level by $\Phi_{s *}=\exp (s \hat{S})$. Using any symbolic algebra package, one computes that the automorphism $\Phi_{s *}$ sends

$$
\begin{aligned}
\hat{X}_{1} & \rightarrow \cos (s) \hat{X}_{1}-\sin (s) \hat{X}_{4}, \\
\hat{X}_{2} & \rightarrow \cos (2 s) \hat{X}_{2}+\sin (2 s) \hat{X}_{3}, \\
\hat{X}_{3} & \rightarrow-\sin (2 s) \hat{X}_{2}+\cos (2 s) \hat{X}_{3}, \\
\hat{X}_{4} & \rightarrow \sin (s) \hat{X}_{1}+\cos (s) \hat{X}_{4}, \\
\hat{Z}_{1} & \rightarrow \cos (s) \hat{Z}_{1}+\sin (s) \hat{Z}_{2}-(1-\cos (s)) \mathcal{Z}, \\
\hat{Z}_{2} & \rightarrow-\sin (s) \hat{Z}_{1}+\cos (s) \hat{Z}_{2}-\sin (s) \mathcal{Z}, \\
\mathcal{Z} & \rightarrow \mathcal{Z} .
\end{aligned}
$$


Let $\hat{\Gamma}$ be any cocompact, discrete subgroup of $\hat{G}$ and let $\hat{\Gamma}_{s}=\Phi_{s}(\hat{\Gamma})$. Then by Corollary 3.10, $\left(\hat{\Gamma}_{s} \backslash \hat{G}, \hat{g}\right)=\left(\hat{\Gamma} \backslash \hat{G}, \Phi_{s}^{*} \hat{g}\right)$ is an isospectral deformation that is not Gordon-Wilson. Theorem 6.1 below shows that $1-\operatorname{spec}\left(\Phi_{s}(\hat{\Gamma}) \backslash \hat{G}, \hat{g}\right)$ is nonconstant, providing an alternate proof that the deformation is not through almost inner automorphisms.

By factoring out the orthogonal matrices as in (3.5), the left invariant metric $\Phi_{s}^{*} \hat{g}$ is determined by the orthonormal basis $\left\{\hat{X}_{1}, \hat{X}_{2}, \hat{X}_{3}, \hat{X}_{4}, \hat{Z}_{1}+(1-\right.$ $\left.\cos (s)) \mathcal{Z}, \hat{Z}_{2}+\sin (s) \mathcal{Z}, \mathcal{Z}\right\}$ of $\hat{\mathfrak{g}}$.

\section{Example II.}

Let

$$
\omega=\alpha_{2} \wedge \alpha_{3}+\alpha_{2} \wedge \alpha_{4}+\alpha_{1} \wedge \zeta_{1}-\alpha_{4} \wedge \zeta_{2}
$$

The closed, nondegenerate two-form $\omega$ induces a strictly nonsingular, threestep nilpotent Lie algebra $\hat{\mathfrak{g}}=\mathfrak{g} \oplus \mathbb{R} \mathcal{Z}$, with Lie bracket and left invariant metric as defined in (3.7). So $\mathfrak{g}$ is the 7-dimensional Lie algebra with orthonormal basis $\left\{\hat{X}_{1}, \hat{X}_{2}, \hat{X}_{3}, \hat{X}_{4}, \hat{Z}_{1}, \hat{Z}_{2}, \mathcal{Z}\right\}$, and Lie bracket

$$
\begin{aligned}
& {\left[\hat{X}_{1}, \hat{X}_{2}\right]=\left[\hat{X}_{3}, \hat{X}_{4}\right]=\hat{Z}_{1},} \\
& {\left[\hat{X}_{1}, \hat{X}_{3}\right]=\hat{Z}_{2},\left[\hat{X}_{4}, \hat{X}_{2}\right]=\hat{Z}_{2}-\mathcal{Z},} \\
& {\left[\hat{X}_{2}, \hat{X}_{3}\right]=\left[\hat{X}_{1}, \hat{Z}_{1}\right]=\left[\hat{Z}_{2}, \hat{X}_{4}\right]=\mathcal{Z},}
\end{aligned}
$$

and all other basis brackets zero.

One easily checks that the derivations $S: \mathfrak{g} \rightarrow \mathfrak{g}$ defined by

$X_{1} \rightarrow-X_{4} ; \quad X_{2} \rightarrow 2 X_{3} ; \quad X_{3} \rightarrow-2 X_{2} ; \quad X_{4} \rightarrow X_{1} ; \quad Z_{1} \rightarrow Z_{2} ; \quad Z_{2} \rightarrow-Z_{1} ;$

and $A: \mathfrak{g} \rightarrow \mathfrak{g}$ defined by

$$
X_{1}, X_{4}, Z_{1}, Z_{2} \rightarrow 0 ; \quad X_{2} \rightarrow-Z_{1} ; \quad X_{3} \rightarrow 2 Z_{2}
$$

satisfy $S^{*} \omega=\alpha_{1} \wedge \alpha_{2}-2 \alpha_{3} \wedge \alpha_{4}$, and $A^{*} \omega=-S^{*} \omega$. Note that $S^{*} \omega$ is not in $\operatorname{span}_{\mathbb{R}}\left\{d \zeta_{1}, d \zeta_{2}, d \xi\right\}$, hence not exact. So neither $S$ nor $A$ will extend to a derivation on $\hat{\mathfrak{g}}$. However, the sum $D=A+S$ is exact as $D^{*} \omega=0$, so $D$ extends to the derivation $\hat{D}: \hat{\mathfrak{g}} \rightarrow \hat{\mathfrak{g}}$ given by $\hat{D}(\hat{X})=(S(X)+A(X), 0)$, $\hat{D}(\mathcal{Z})=0$. That is, $\hat{D}$ sends

$$
\begin{gathered}
\hat{X}_{1} \rightarrow-\hat{X}_{4} ; \quad \hat{X}_{2} \rightarrow 2 \hat{X}_{3}-\hat{Z}_{1} ; \quad \hat{X}_{3} \rightarrow-2 \hat{X}_{2}+2 \hat{Z}_{2} ; \quad \hat{X}_{4} \rightarrow \hat{X}_{1} ; \\
\hat{Z}_{1} \rightarrow \hat{Z}_{2} ; \quad \hat{Z}_{2} \rightarrow-\hat{Z}_{1} ; \quad \mathcal{Z} \rightarrow 0 .
\end{gathered}
$$

Let $\hat{G}$ denote the simply connected, strictly nonsingular nilpotent Lie group with Lie algebra $\hat{\mathfrak{g}}$. Define a continuous family $\Phi_{s}$ of Lie group automorphisms 
of $\hat{G}$ defined on the Lie algebra level by $\Phi_{s *}=\exp (s \hat{D})$. The automorphism $\Phi_{s *}$ sends

$$
\begin{aligned}
\hat{X}_{1} & \rightarrow \cos (s) \hat{X}_{1}-\sin (s) \hat{X}_{4}, \\
\hat{X}_{2} & \rightarrow \cos (2 s) \hat{X}_{2}+\sin (2 s) \hat{X}_{3}-\sin (s) \hat{Z}_{1}+(\cos (s)-\cos (2 s)) \hat{Z}_{2}, \\
\hat{X}_{3} & \rightarrow-\sin (2 s) \hat{X}_{2}+\cos (2 s) \hat{X}_{3}+\sin (2 s) \hat{Z}_{2}, \\
\hat{X}_{4} & \rightarrow \sin (s) \hat{X}_{1}+\cos (s) \hat{X}_{4}, \\
\hat{Z}_{1} & \rightarrow \cos (s) \hat{Z}_{1}+\sin (s) \hat{Z}_{2}, \\
\hat{Z}_{2} & \rightarrow-\sin (s) \hat{Z}_{1}+\cos (s) \hat{Z}_{2}, \\
\mathcal{Z} & \rightarrow \mathcal{Z} .
\end{aligned}
$$

Let $\hat{\Gamma}$ be any cocompact, discrete subgroup of $\hat{G}$. Then $\left(\Phi_{s}(\hat{\Gamma}) \backslash \hat{G}, \hat{g}\right)=$ $\left(\hat{\Gamma} \backslash \hat{G}, \Phi_{s}^{*} \hat{g}\right)$ is an isospectral deformation not arising from almost inner automorphisms. We show in Theorem 6.2 below that $1-\operatorname{spec}\left(\Phi_{s}(\hat{\Gamma}) \backslash \hat{G}, \hat{g}\right)$ is nonconstant, providing an alternate proof that the deformation is not Gordon-Wilson.

By factoring out the orthogonal matrices as in (3.5), we see that $\Phi_{s}^{*} \hat{g}$ is determined by the orthonormal basis $\left\{\hat{X}_{1}, \hat{X}_{2}+\sin (s) \cos (2 s) \hat{Z}_{1}-(1-\right.$ $\left.\cos (s) \cos (2 s)) \hat{Z}_{2}, \hat{X}_{3}-\sin (s) \sin (2 s) \hat{Z}_{1}-\cos (s) \sin (2 s) \hat{Z}_{2}, \hat{X}_{4}, \hat{Z}_{1}, \hat{Z}_{2}, \mathcal{Z}\right\}$ of $\hat{\mathfrak{g}}$.

\section{Section 5. The Marked Length Spectrum.}

In this section we prove the following.

5.1 Theorem. The nilmanifolds $\left(\hat{\Gamma}_{s} \backslash \hat{G}, \hat{g}\right)$ presented in Example I above have the same marked length spectrum for all s. In particular, $\Phi_{s}$ marks the length spectrum from $\left(\hat{\Gamma}_{0} \backslash \hat{G}, \hat{g}\right)$ to $\left(\hat{\Gamma}_{s} \backslash \hat{G}, \hat{g}\right)$.

5.2 Theorem. The nilmanifolds $\left(\hat{\Gamma}_{s} \backslash \hat{G}, \hat{g}\right)$ presented in Example II above have the same marked length spectrum for all $s$. In particular, $\Phi_{s}$ marks the length spectrum from $\left(\hat{\Gamma}_{0} \backslash \hat{G}, \hat{g}\right)$ to $\left(\hat{\Gamma}_{s} \backslash \hat{G}, \hat{g}\right)$.

5.3 Remark. These are the first continuous families of Riemannian manifolds with the same marked length spectrum but not the same spectrum on 1-forms. Discrete families of manifolds with these properties were presented in [Gt4]. The only other known examples of manifolds that have the same marked length spectrum but not the same 1-form spectrum are the Zoll spheres and standard spheres, which are not even isospectral on functions.

If $G$ is a simply connected Lie group with cocompact, discrete subgroup $\Gamma$, then $\Gamma$ is also the fundamental group of $\Gamma \backslash G$, so free homotopy classes of loops of $\Gamma \backslash G$ correspond to conjugacy classes in $\Gamma$. We denote by $[\gamma]_{\Gamma}$ the conjugacy class in $\Gamma$ represented by $\gamma \in \Gamma$, that is, $[\gamma]_{\Gamma}=\left\{\gamma^{\prime} \gamma \gamma^{-1}: \gamma^{\prime} \in \Gamma\right\}$. Recall that any isomorphism between fundamental groups induces a correspondence between free homotopy classes of loops. 
5.4 Definition. A pair of Riemannian manifolds $\left(\Gamma_{1} \backslash \tilde{M}_{1}, g_{1}\right)$ and $\left(\Gamma_{2} \backslash \tilde{M}_{2}, g_{2}\right)$ have the same marked length spectrum if and only if there exists an isomorphism $\Phi: \Gamma_{1} \rightarrow \Gamma_{2}$ between their fundamental groups such that corresponding free homotopy classes contain closed geodesics of the same length. That is, the free homotopy class represented by $[\gamma]_{\Gamma_{1}}$ contains a closed geodesic of (positive) period $\lambda$ if and only if the free homotopy class represented by $[\Phi(\gamma)]_{\Gamma_{2}}$ also contains a closed geodesic of period $\lambda$. We say that $\Phi$ marks the length spectrum from $\left(\Gamma_{1} \backslash \tilde{M}_{1}, g_{1}\right)$ to $\left(\Gamma_{2} \backslash \tilde{M}_{2}, g_{2}\right)$.

We have the following sufficient condition for a pair of nilmanifolds to have the same marked length spectrum.

5.5 Theorem [G1, Theorem 1.8]. Let $G$ be a simply connected nilpotent Lie group with cocompact, discrete subgroup $\Gamma$. Let $\Phi$ be a $\Gamma$-almost inner automorphism of $G$. Then for any left invariant metric $g$ of $G, \Phi$ marks the length spectrum from $(\Gamma \backslash G, g)$ to $(\Phi(\Gamma) \backslash G, g)$.

Trivially, if $\Phi$ is an orthogonal automorphism, i.e., $\Phi \in \mathrm{OA}(G, g)$, then $\Phi$ marks the length spectrum from $(\Gamma \backslash G, g)$ to $(\Phi(\Gamma) \backslash G, g)$. Conversely, on two-step nilmanifolds Eberlein showed the following.

5.6 Theorem [E, Theorem 5.20]. Let $\Gamma_{1}, \Gamma_{2}$ be cocompact, discrete subgroups of simply connected, two-step nilpotent Lie groups $G_{1}, G_{2}$ with left invariant metrics $g_{1}, g_{2}$ respectively. Assume that $\left(\Gamma_{1} \backslash G_{1}, g_{1}\right)$ and $\left(\Gamma_{2} \backslash G_{2}, g_{2}\right)$ have the same marked length spectrum, and let $\Phi: \Gamma_{1} \rightarrow \Gamma_{2}$ be an isomorphism inducing this marking. Then $\Phi=\left.\left(\Phi_{1} \circ \Phi_{2}\right)\right|_{\Gamma_{1}}$, where $\Phi_{2}$ is a $\Gamma_{1}$-almost inner automorphism of $G_{1}$, and $\Phi_{1}$ is an isomorphism of $\left(G_{1}, g_{1}\right)$ onto $\left(G_{2}, g_{2}\right)$ that is also an isometry. Moreover, this factorization is unique. In particular, if $\left(\Gamma_{1} \backslash G_{1}, g_{1}\right)$ and $\left(\Gamma_{2} \backslash G_{2}, g_{2}\right)$ have the same marked length spectrum, they necessarily have the same spectrum of the Laplacian on functions and on $p$ forms for all $p$.

In contrast to (5.6), we have the following necessary and sufficient condition for a large family of pairs of three-step nilmanifolds to have the same marked length spectrum.

5.7 Theorem [Gt4, Theorem 3.3.1]. Let $G$ be a simply connected, strictly nonsingular, three-step nilpotent Lie group with a one-dimensional center. Let $\Gamma_{1}$ and $\Gamma_{2}$ be cocompact, discrete subgroups of $G$ such that $\Gamma_{1} \cap Z(G)=\Gamma_{2} \cap Z(G)$. Let $g$ be any left invariant metric on $G$. Then $\left(\Gamma_{1} \backslash G, g\right)$ and $\left(\Gamma_{2} \backslash G, g\right)$ have the same marked length spectrum if and only if there exists an isomorphism $\Phi: \Gamma_{1} \rightarrow \Gamma_{2}$ such that $\bar{\Phi}: \bar{\Gamma}_{1} \rightarrow \bar{\Gamma}_{2}$ marks the length spectrum between $\left(\bar{\Gamma}_{1} \backslash \bar{G}, \bar{g}\right)$ and $\left(\bar{\Gamma}_{2} \backslash \bar{G}, \bar{g}\right)$.

Proof of 5.1 and 5.2. The automorphisms $\Phi_{s}$ presented in Example I and Example II have the property that $\bar{\Phi}_{s}$ is the composition of an almost inner automorphism and an orthogonal automorphism, which by (5.5) necessarily 
mark the length spectrum from $\left(\Gamma_{0} \backslash G, g\right)$ to $\left(\Gamma_{s} \backslash G, g\right)$. The result now follows from (5.7).

We also showed in [Gt4] that for a large class of three-step nilmanifolds, which include Example I and Example II, if a family of nilmanifolds in this class have the same marked length spectrum, they necessarily share the same Laplace spectrum on functions, providing an alternate proof that Example I and Example II are isospectral deformations.

5.8 Theorem [Gt4, Theorem 3.3.2]. Let $G$ be a simply connected, strictly nonsingular, three-step nilpotent Lie group. Let $\Gamma_{1}$ and $\Gamma_{2}$ be cocompact, discrete subgroups of $G$ such that $\Gamma_{1} \cap Z(G)=\Gamma_{2} \cap Z(G)$. If $\left(\Gamma_{1} \backslash G, g\right)$ and $\left(\Gamma_{2} \backslash G, g\right)$ have the same marked length spectrum, then $\left(\Gamma_{1} \backslash G, g\right)$ and $\left(\Gamma_{2} \backslash G, g\right)$ are isospectral on functions.

\section{Section 6: Comparing the 1-form spectrum of nilmanifolds.}

In this section, we prove the following.

6.1 Theorem. The nilmanifolds $\left(\hat{\Gamma}_{s} \backslash \hat{G}, \hat{g}\right)$ of Example I are not isospectral on 1-forms.

6.2 Theorem. The nilmanifolds $\left(\hat{\Gamma}_{s} \backslash \hat{G}, \hat{g}\right)$ of Example II are not isospectral on 1-forms.

Proof of Theorem 6.1.

Let $\hat{G}, \hat{\mathfrak{g}}, \hat{g}$, and $\Phi_{s}$ be as in Example I, Section 4 . Let $\hat{\Gamma}$ be an arbitrary cocompact, discrete subgroup of $\hat{G}$, and let $\hat{\Gamma}_{s}=\Phi_{s}(\hat{\Gamma})$. We calculate a monic polynomial $P_{s}(x)$ of degree 7 whose roots must appear in 1 -spec $\left(\hat{\Gamma}_{s} \backslash \hat{G}, \hat{g}\right)$. We show that the coefficients of $P_{s}(x)$, which must change continuously with $s$, are nonconstant. The result follows.

We refer the reader to [Gt3, Appendix] for definitions, notation, and an exposition of the method used below to demonstrate the inequality of the 1form spectrum. We include only those details needed for verification.

The main tool we use is representation theory. Irreducible representations of nilpotent Lie groups are well understood due to Kirillov theory. In particular, the irreducible representations of a nilpotent Lie group $G$ correspond to the coadjoint orbits of the Lie algebra $\mathfrak{g}$ of $G$. For $\tau \in \mathfrak{g}^{*}$, we denote by $\pi_{\tau}$ the corresponding irreducible representation of $G$. A good reference for representations of nilpotent Lie groups is [CG].

We use only the characters of $\hat{G}$, i.e., the (complex) one-dimensional irreducible representations to demonstrate the inequality of the 1-form spectrum. The dimension of the representation space $\mathcal{H}_{\tau}$ of $\pi_{\tau}$ corresponds to the dimension of the coadjoint orbit of $\tau$. In particular, the one-dimensional representations of $G$ correspond to those elements of $\mathfrak{g}^{*}$ that are zero on the derived algebra $[\mathfrak{g}, \mathfrak{g}]$ of $\mathfrak{g}$. 
Consider $\rho_{\hat{\Gamma}_{s}}$, the quasi-regular representation of $\hat{G}$ on $L^{2}\left(\hat{\Gamma}_{s} \backslash \hat{G}\right)$. For $f \in$ $L^{2}\left(\hat{\Gamma}_{s} \backslash \hat{G}\right)$, and $x \in \hat{G}, \rho_{\hat{\Gamma}_{s}}(x) f=f \circ R_{x}$, where $R$ denotes the right action of $\hat{G}$ on $\hat{\Gamma}_{s} \backslash \hat{G}$. It is known that $L^{2}\left(\hat{\Gamma}_{s} \backslash \hat{G}\right)$ is completely reducible as

$$
L^{2}\left(\hat{\Gamma}_{s} \backslash \hat{G}\right) \cong \bigoplus_{\tau \in \mathcal{T}_{s}} m_{s}(\tau) \mathcal{H}_{\tau}
$$

Here $m_{s}(\tau)$ is the multiplicity of $\pi_{\tau}$ in $\rho_{\hat{\Gamma}_{s}}$, and $\mathcal{T}_{s}$ is a subset of $\hat{\mathfrak{g}}^{*}$ containing at most one element of each coadjoint orbit of $\hat{\mathfrak{g}}^{*}$.

Let $\left\{\alpha_{1}, \alpha_{2}, \alpha_{3}, \alpha_{4}, \zeta_{1}, \zeta_{2}, \xi\right\}$ be the dual basis to the orthonormal basis $\left\{\hat{X}_{1}\right.$, $\left.\hat{X}_{2}, \hat{X}_{3}, \hat{X}_{4}, \hat{Z}_{1}, \hat{Z}_{2}, \mathcal{Z}\right\}$ of $\hat{\mathfrak{g}}$. Denote by $\mathfrak{C}$ the subspace $\mathfrak{C}=\operatorname{span}_{\mathbb{R}}\left\{\alpha_{1}, \alpha_{2}, \alpha_{3}, \alpha_{4}\right\}$ of $\hat{\mathfrak{g}}^{*}$. Note that $\mathfrak{C}=\left\{\tau \in \hat{\mathfrak{g}}^{*}: \tau([\hat{\mathfrak{g}}, \hat{\mathfrak{g}}]) \equiv 0\right\}$. Thus the characters of $\hat{G}$ appearing in $L^{2}\left(\hat{\Gamma}_{s} \backslash \hat{G}\right)$ correspond to $\mathcal{T}_{s} \cap \mathfrak{C}$.

Let $\tau \in \mathfrak{C}$. We now calculate $\Delta$ acting on $\mathcal{H}_{\tau} \otimes \Lambda^{1}\left(\hat{\mathfrak{g}}^{*}\right)$, an invariant subspace of $\Delta$. Note that if $\mathbb{C} \Lambda^{1}\left(\hat{\mathfrak{g}}^{*}\right)$ denotes $\Lambda^{1}\left(\hat{\mathfrak{g}}^{*}\right)$ with complex coefficients, then $\mathcal{H}_{\tau} \otimes \Lambda^{1}\left(\hat{\mathfrak{g}}^{*}\right)=F_{\tau} \otimes \mathbb{C} \Lambda^{1}\left(\hat{\mathfrak{g}}^{*}\right)$, where $F_{\tau}(x)=e^{(2 \pi i \tau(\log (x)))}$.

As $\hat{Z}_{1} F_{\tau}=\hat{Z}_{2} F_{\tau}=\mathcal{Z} F_{\tau}=0$, we know $\Delta F_{\tau}=4 \pi^{2}\|\tau\|^{2} F_{\tau}$. One also computes the following.

$\Delta \alpha_{1}=\Delta \alpha_{2}=\Delta \alpha_{3}=\Delta \alpha_{4}=0, \quad \Delta \zeta_{1}=2 \zeta_{1}+2 \xi, \quad \Delta \zeta_{2}=2 \zeta_{2}, \quad \Delta \xi=2 \zeta_{1}+5 \xi$

\begin{tabular}{|c|c|c|c|c|c|c|c|}
\hline$\nabla_{U} \mu$ & $\alpha_{1}$ & $\alpha_{2}$ & $\alpha_{3}$ & $\alpha_{4}$ & $\zeta_{1}$ & $\zeta_{2}$ & $\xi$ \\
\hline$X_{1}$ & 0 & $\frac{1}{2}\left(\zeta_{1}+\xi\right)$ & $\frac{1}{2} \zeta_{2}$ & 0 & $\frac{1}{2}\left(-\alpha_{2}+\xi\right)$ & $-\frac{1}{2} \alpha_{3}$ & $-\frac{1}{2}\left(\alpha_{2}+\zeta_{1}\right)$ \\
\hline$X_{2}$ & $-\frac{1}{2}\left(\zeta_{1}+\xi\right)$ & 0 & $\frac{1}{2} \xi$ & $-\frac{1}{2} \zeta_{2}$ & $\frac{1}{2} \alpha_{1}$ & $\frac{1}{2} \alpha_{4}$ & $\frac{1}{2}\left(\alpha_{1}-\alpha_{3}\right)$ \\
\hline$X_{3}$ & $-\frac{1}{2} \zeta_{2}$ & $-\frac{1}{2} \xi$ & 0 & $\frac{1}{2}\left(\zeta_{1}+\xi\right)$ & $-\frac{1}{2} \alpha_{4}$ & $\frac{1}{2} \alpha_{1}$ & $\frac{1}{2}\left(\alpha_{2}-\alpha_{4}\right)$ \\
\hline$X_{4}$ & 0 & $\frac{1}{2} \zeta_{2}$ & $-\frac{1}{2}\left(\zeta_{1}+\xi\right)$ & 0 & $\frac{1}{2} \alpha_{3}$ & $-\frac{1}{2}\left(\alpha_{2}+\xi\right)$ & $\frac{1}{2}\left(\alpha_{3}+\zeta_{2}\right)$ \\
\hline
\end{tabular}

Write $\tau=A_{1} \alpha_{1}+A_{2} \alpha_{2}+A_{3} \alpha_{3}+A_{4} \alpha_{4}$ where $A_{i} \in \mathbb{R}$. Using $\Delta(f \tau)=$ $(\Delta f) \tau+f(\Delta \tau)-2 \nabla_{\operatorname{grad} f} \tau$ and the above information, a straightforward calculation shows that if we set $E_{\tau}=$

$\left(\begin{array}{ccccccc}4 \pi^{2}\|\tau\|^{2} & 0 & 0 & 0 & 2 \pi i A_{2} & 2 \pi i A_{3} & 2 \pi i A_{2} \\ 0 & 4 \pi^{2}\|\tau\|^{2} & 0 & 0 & -2 \pi i A_{1} & -2 \pi i A_{4} & 2 \pi i\left(A_{3}-A_{1}\right) \\ 0 & 0 & 4 \pi^{2}\|\tau\|^{2} & 0 & 2 \pi i A_{4} & -2 \pi i A_{1} & 2 \pi i\left(A_{4}-A_{2}\right) \\ 0 & 0 & 0 & 4 \pi^{2}\|\tau\|^{2} & -2 \pi i A_{3} & 2 \pi i A_{2} & -2 \pi i A_{3} \\ -2 \pi i A_{2} & 2 \pi i A_{1} & -2 \pi i A_{4} & 2 \pi i A_{3} & 4 \pi^{2}\|\tau\|^{2}+2 & 0 & -2 \pi i A_{1}+2 \\ -2 \pi i A_{3} & 2 \pi i A_{4} & 2 \pi i A_{1} & -2 \pi i A_{2} & 0 & 4 \pi^{2}\|\tau\|^{2}+2 & 2 \pi i A_{4} \\ -2 \pi i A_{2} & 2 \pi i\left(A_{1}-A_{3}\right) & 2 \pi i\left(A_{2}-A_{4}\right) & 2 \pi i A_{3} & 2 \pi i A_{1}+2 & -2 \pi i A_{4} & 4 \pi^{2}\|\tau\|^{2}+5\end{array}\right)$


then there exists $\mu \in \mathbb{C} \Lambda^{1}\left(\hat{\mathfrak{g}}^{*}\right)$, such that $\Delta\left(F_{\tau} \otimes \mu\right)=\lambda\left(F_{\tau} \otimes \mu\right)$ if and only if $\lambda$ is an eigenvalue of the matrix $E_{\tau}$. That is, for every $\tau \in \mathcal{T}_{s} \cap \mathfrak{C}$, the roots of the characteristic polynomial of $E_{\tau}$ are contained in $1-\operatorname{spec}\left(\Gamma_{s} \backslash G, g\right)$. One easily computes that $\tau \in \mathcal{T}_{s} \cap \mathfrak{C}$ if and only if $\tau\left(\log \hat{\Gamma}_{s}\right) \subset \mathbb{Z}$.

Now $\log \hat{\Gamma}_{s}$ generates a lattice of full rank in $\hat{\mathfrak{g}}$, so we can find $V_{1}^{s}, V_{2}^{s}, V_{3}^{s}$, $V_{4}^{s}$, elements of $\operatorname{span}_{\mathbb{R}}\left\{\hat{X}_{1}, \hat{X}_{2}, \hat{X}_{3}, \hat{X}_{4}\right\}$, with the property that $\tau\left(\log \hat{\Gamma}_{s}\right) \subset \mathbb{Z}$ if and only if $\tau\left(V_{i}^{s}\right) \subset \mathbb{Z}, i=1,2,3,4$. Let $L_{s}=\operatorname{span}_{\mathbb{Z}}\left\{V_{1}^{s}, V_{2}^{s}, V_{3}^{s}, V_{4}^{s}\right\}$. Note that $L_{s}$ is a lattice of full rank in $\operatorname{span}_{\mathbb{R}}\left\{\hat{X}_{1}, \hat{X}_{2}, \hat{X}_{3}, \hat{X}_{4}\right\}$. Let $L_{s}^{*}$ be the dual lattice of $L_{s}$ in $\mathfrak{C}$, so $L_{s}^{*}$ is a lattice of full rank in $\mathfrak{C}$, and

$$
\tau \in \mathcal{T}_{s} \cap \mathfrak{C} \text { if and only if } \tau \in L_{s}^{*} .
$$

A straightforward computation shows that if $\tau=a_{1} \alpha_{1}+a_{2} \alpha_{2}+a_{3} \alpha_{3}+a_{4} \alpha_{4}$ is any element of $L_{0}^{*} \subset \mathcal{T}_{0} \cap \mathfrak{C}$ then $\tau_{s}=\tau \circ \Phi_{s *}^{-1}$ is an element of $L_{s}^{*} \subset \mathcal{T}_{s} \cap \mathfrak{C}$. That is, $\tau_{s}=A_{1}(s) \alpha_{1}+A_{2}(s) \alpha_{2}+A_{3}(s) \alpha_{3}+A_{4}(s) \alpha_{4}$ is an element of $L_{s}^{*}$, where

$$
\begin{aligned}
& A_{1}(s)=a_{1} \cos (s)+a_{4} \sin (s) \\
& A_{2}(s)=a_{2} \cos (2 s)-a_{3} \sin (2 s) \\
& A_{3}(s)=a_{2} \sin (2 s)+a_{3} \cos (2 s) \\
& A_{4}(s)=-a_{1} \sin (s)+a_{4} \cos (s) .
\end{aligned}
$$

Note that $\left\|\tau_{s}\right\|^{2}=a_{1}^{2}+a_{2}^{2}+a_{3}^{2}+a_{4}^{2}$ is independent of $s$. Using any symbolic computation package, one easily checks that the characteristic polynomial of $E_{\tau_{s}}$, denoted $P_{s}(x)$, is a monic polynomial of degree 7 , and the coefficients of the monic polynomial $P_{s}(x)$ are independent of $s$ if and only if $a_{1} a_{2}=a_{3} a_{4}$. As $L_{0}^{*}$ is full lattice in $\mathfrak{C}$, there exists at least one $\tau \in L_{0}^{*}$ satisfying $a_{1} a_{2} \neq a_{3} a_{4}$, and we are done.

6.3 Remark. Alternatively, one may perform the same computation by viewing the manifolds of Example I as $\left(\hat{\Gamma} \backslash \hat{G}, \Phi_{s}^{*} \hat{g}\right)$. This is simplified by factoring out an orthogonal matrix for each $s$, as in (3.5), obtaining the orthonormal basis $\left\{\hat{X}_{1}, \hat{X}_{2}, \hat{X}_{3}, \hat{X}_{4}, \hat{Z}_{1}+(1-\cos (s)) \mathcal{Z}, \hat{Z}_{2}+\sin (s) \mathcal{Z}, \mathcal{Z}\right\}$ of $\hat{\mathfrak{g}}$. Here the functional $\tau$ remains fixed, but the values for $\Delta \alpha_{i}, \Delta \zeta_{i}$ and $\nabla_{U} \mu$ listed above now vary with $s$. One obtains a different expression for $E_{\tau}$ depending on $s$, but the characteristic polynomial $P_{s}(x)$ is equal to that obtained above.

Proof of Theorem 6.2.

Let $\hat{G}, \hat{\mathfrak{g}}, \hat{g}$, and $\Phi_{s}$ be as in Example II, Section 4 . Let $\hat{\Gamma}$ be an arbitrary cocompact, discrete subgroup of $\hat{G}$, and let $\hat{\Gamma}_{s}=\Phi_{s}(\hat{\Gamma})$. Let $\left\{\alpha_{1}, \alpha_{2}, \alpha_{3}, \alpha_{4}, \zeta_{1}, \zeta_{2}, \xi\right\}$ be the dual basis to the orthonormal basis $\left\{\hat{X}_{1}, \hat{X}_{2}, \hat{X}_{3}, \hat{X}_{4}, \hat{Z}_{1}, \hat{Z}_{2}, \mathcal{Z}\right\}$ of $\hat{\mathfrak{g}}$.

The proof here is identical to that of Example I, with the following modifications.

$\Delta \alpha_{1}=\Delta \alpha_{2}=\Delta \alpha_{3}=\Delta \alpha_{4}=0, \quad \Delta \zeta_{1}=2 \zeta_{1}, \quad \Delta \zeta_{2}=2 \zeta_{2}-\xi, \quad \Delta \xi=-\zeta_{1}+4 \xi$. 


\begin{tabular}{|c|c|c|c|c|c|c|c|}
\hline$\nabla_{U} \mu$ & $\alpha_{1}$ & $\alpha_{2}$ & $\alpha_{3}$ & $\alpha_{4}$ & $\zeta_{1}$ & $\zeta_{2}$ & $\xi$ \\
\hline$X_{1}$ & 0 & $\frac{1}{2} \zeta_{1}$ & $\frac{1}{2} \zeta_{2}$ & 0 & $\frac{1}{2}\left(-\alpha_{2}+\xi\right)$ & $-\frac{1}{2} \alpha_{3}$ & $-\frac{1}{2} \zeta_{1}$ \\
\hline$X_{2}$ & $-\frac{1}{2} \zeta_{1}$ & 0 & $\frac{1}{2} \xi$ & $\frac{1}{2}\left(-\zeta_{2}+\xi\right)$ & $\frac{1}{2} \alpha_{1}$ & $\frac{1}{2} \alpha_{4}$ & $-\frac{1}{2}\left(\alpha_{3}+\alpha_{4}\right)$ \\
\hline$X_{3}$ & $-\frac{1}{2} \zeta_{2}$ & $-\frac{1}{2} \xi$ & 0 & $\frac{1}{2} \zeta_{1}$ & $-\frac{1}{2} \alpha_{4}$ & $\frac{1}{2} \alpha_{1}$ & $\frac{1}{2} \alpha_{2}$ \\
\hline$X_{4}$ & 0 & $\frac{1}{2}\left(\zeta_{2}-\xi\right)$ & $-\frac{1}{2} \zeta_{1}$ & 0 & $\frac{1}{2} \alpha_{3}$ & $-\frac{1}{2}\left(\alpha_{2}+\xi\right)$ & $\frac{1}{2}\left(\alpha_{2}+\zeta_{2}\right)$ \\
\hline
\end{tabular}

Set $E_{\tau}=$

$\left(\begin{array}{ccccccc}4 \pi^{2}\|\tau\|^{2} & 0 & 0 & 0 & 2 \pi i A_{2} & 2 \pi i A_{3} & 0 \\ 0 & 4 \pi^{2}\|\tau\|^{2} & 0 & 0 & -2 \pi i A_{1} & -2 \pi i A_{4} & 2 \pi i\left(A_{3}+A_{4}\right) \\ 0 & 0 & 4 \pi^{2}\|\tau\|^{2} & 0 & 2 \pi i A_{4} & -2 \pi i A_{1} & -2 \pi i A_{2} \\ 0 & 0 & 0 & 4 \pi^{2}\|\tau\|^{2} & -2 \pi i A_{3} & 2 \pi i A_{2} & -2 \pi i A_{2} \\ -2 \pi i A_{2} & 2 \pi i A_{1} & -2 \pi i A_{4} & 2 \pi i A_{3} & 4 \pi^{2}\|\tau\|^{2}+2 & 0 & -2 \pi i A_{1} \\ -2 \pi i A_{3} & 2 \pi i A_{4} & 2 \pi i A_{1} & -2 \pi i A_{2} & 0 & 4 \pi^{2}\|\tau\|^{2}+2 & 2 \pi i A_{4}-1 \\ 0 & -2 \pi i\left(A_{3}+A_{4}\right) & 2 \pi i A_{2} & 2 \pi i A_{2} & 2 \pi i A_{1} & -2 \pi i A_{4}-1 & 4 \pi^{2}\|\tau\|^{2}+4\end{array}\right)$

We may use the same values for $A_{i}(s), i=1,2,3,4$.

\section{REFERENCES}

[B1] P. Bérard, Spectral Geometry: Direct and Inverse Problems, Lecture Notes in Mathematics, vol. 1207, Springer-Verlag, New York, 1980.

[B2] ㄴ. Variéteś Riemanniennes isospectrales non isométriques, Astérisque 177178 (1989), 127-154.

[BG] R. Brooks and C. S. Gordon, Isospectral families of conformally equivalent Riemannian metrics, Bull. Amer. Math. Soc. (N.S.) 23 (1990), 433-436.

[C] I. Chavel, Eigenvalues in Riemannian Geometry, Acad. Press, 1984.

[CdV] Y. Colin de Verdière, Spectre du laplacien et longueurs des géodésiques périodiques, I-II, Compositio Math 27 (1973), 83-106, 159-184.

[CG] L. Corwin and F. P. Greenleaf, Representations of Nilpotent Lie Groups and Their Applications; Part 1: Basic Theory and Examples, Cambridge University Press, New York, 1990.

[CS] C. Croke and V. Sharafutdinov, Spectral rigidity of a compact negatively curved manifold, preprint (1996).

[Cr] C. Croke, Rigidity for surfaces of non-negative curvature, Comment. Math. Helvetici 65 (1990), 150-169.

[DGGW1] D. DeTurck, H. Gluck, C. S. Gordon, D. Webb, You cannot hear the mass of a homology class, Comment. Math. Helvetici 64 (1989), 589-617.

[DGGW2] ㄴ. The geometry of isospectral deformations, Proc. of Symposia in Pure Math. 54 (1993), 135-154. 
CONTINUOUS FAMILIES OF ISOSPECTRAL RIEMANNIAN MANIFOLDS. 19

[DGGW3] _ , The inaudible geometry of nilmanifolds, Invent. Math. 111 (1993), 271284.

[DG1] D. DeTurck and C. S. Gordon, Isospectral Deformations I: Riemannian structures on two-step nilspaces, Comm. Pure Appl. Math. 40 (1987), 367-387.

[DG2] _ Isospectral Deformations II: trace formulas, metrics, and potentials, Comm. Pure Appl. Math. 42 (1989), 1067-1095.

[DGu] J. J. Duistermaat and V. W. Guillemin, The spectrum of poitive elliptic operators and periodic bicharacteristics, Invent. Math. 29 (1977), 39-79.

[E] P. Eberlein, Geometry of two-step nilpotent groups with a left invariant metric, Ann. Scien. de l'Ecole Norm. Sup. 27 (1994), 611-660.

[G1] C. S. Gordon, The Laplace spectra versus the length spectra of Riemannian manifolds, Contemporary Mathematics 51 (1986), 63-79.

[G2] , Riemannian manifolds isospectral on functions but not on 1-forms, J. Diff. Geom. 24 (1986), 79-96.

[G3] Isospectral closed Riemannian manifolds which are not locally isometric, J. Diff. Geom. 37 (1993), 639-649.

[G4] Isospectral closed Riemannian manifolds which are not locally isometric:II, Contemporary Mathematics: Geometry of the Spectrum (R. Brooks, C. S. Gordon, P. Perry, eds.), vol. 173, AMS, 1994, pp. 121-131.

[GM1] C. S. Gordon and Y. Mao, Comparisons of Laplace spectra, length spectra and geodesic flows of some Riemannian manifolds, Math Research Letter 1 (1994), 677-688.

[GM2] Geodesic conjugacy in 2-step nilmanifolds, preprint (1995).

[GMS] C. S. Gordon, Y. Mao, and D. Schueth, Symplectic rigidity of geodesic flows on two-step nilmanifolds, preprint (1995).

[GW1] C. S. Gordon and E. N. Wilson, Isospectral deformations of compact solvmanifolds, J. Differential Geometry 19 (1984), 241-256.

[GW2] - The spectrum of the Laplacian on Riemannian Heisenberg manifolds, Mich. Math. J. 33 (1986), 253-271.

[GW3] C Continuous families of isospectral Riemannian manifolds which are not locally isometric, preprint (1995).

[Gt1] Ruth Gornet, Equivalence of quasi-regular representations of two and three-step nilpotent Lie groups, J. Fn. An. 119 (1994), 121-137.

[Gt2] _ The length spectrum and representation theory on two and three-step nilpotent Lie groups, Contemporary Mathematics: Geometry of the Spectrum (R. Brooks, C.S. Gordon, P. Perry, eds.), vol. 173, AMS, 1994, pp. 133-156.

[Gt3] A A new construction of isospectral Riemannian nilmanifolds with examples, Mich. Math. J. 43 (1996), 159-188.

[Gt4] - The marked length spectrum vs. the p-form spectrum of Riemannian nilmanifolds, Comm. Math. Helv. 71 (1996), 297-329.

[GuK] V. Guillemin and D. Kazhdan, Proc. Symp. Pure Math., Geometry of the Laplace Operator, vol. 36, Amer. Math. Soc., 1980, pp. 153-180.

[I1] A. Ikeda, Isospectral problem for spherical space forms, in "Spectra of Riemannian Manifolds," (1983), Kaigai Publications, 57-63.

[I2] L Riemannian manifolds $p$-isospectral but not $(p+1)$-isospectral, Geometry of Manifolds (Matsumoto), Perspect. Math., vol. 8, Academic Press, Boston, MA, 1989, pp. 383-417.

[Ot1] J. Otal, Le spectre margué des longeurs des surfaces à courbure négative, Ann. of Math. 131 (1990), 151-162.

[Ot2] _ Sur les longueurs des géodesiques d'une metrique a courbure négative dans le disque, Comm. Math. Helvetici 65 (1990), 334-347. 
[O] He Ouyang, On isospectral deformations on two-step nilmanifolds, PhD Dissertation, Washington University (1991).

[OP] He Ouyang and H. Pesce, Déformations isospectrales sur les nilvariétés de rang deaux, C. R. Acad. Sci. Paris, Série I 314 (1992), 621-623.

[P1] Hubert Pesce, Déformations isospectrales de certaines nilvariétés et finitude spectrale des variétés de Heisenberg, Ann. Sci. École Norm. Sup. 25 (1992), 515-538.

[P2] D Déformations L-isospectrales sur les nilvariétés de rang deux, C. R. Acad. Sci. Paris, Série I 315 (1992), 821-823.

[P3] Calcul du spectre d'une nilvariété de rang deux et applications, Trans. Amer. Math. Soc. 339 (1993), 433-461.

[P4] Variétés isospectrales et representations de groupes, Contemporary Mathematics: Geometry of the Spectrum (R. Brooks, C. S. Gordon, P. Perry, eds.), vol. 173, AMS, 1994, pp. 231-240.

[Sch1] D. Schueth, Continuous families of quasi-regular representations of solvable Lie groups, J. Fn. An. 134 (1995), 247-259.

[Sch2] U Isospectral deformations on Riemannian manifolds which are diffeomorphic to compact Heisenberg manifolds, Comment. Math. Helvetici 70 (1995), 434-454.

[W] E. N. Wilson, Isometry groups on homogeneous nilmanifolds, Geom. Dedicata 12 (1982), 337-346.

[Wp] S. Wolpert, The spectrum as moduli for flat tori, Trans. AMS 244 (1978), 313321.

Ruth Gornet; Texas Tech University; Department of Mathematics; Lubbock, Texas 79409-1042; E-mail: Gornet@math.Ttu.edu_February 1997 